\title{
Associations between regional and whole-body fat and insulin sensitivity in type 2 diabetic men of White and Black ethnicity
}

Differences in regional and whole-body (WB) fat deposition are consistently reported between people of Black and White ethnicity ${ }^{(1)}$. People of Black ethnicity have lower waist circumference (WC), due to less visceral adipose tissue (VAT), despite a 2-3-fold higher prevalence of type 2 diabetes (T2D) compared to Whites ${ }^{(2)}$; thus, an under-diagnosis of the metabolic syndrome, for a given BMI, is common in Black populations compared to other ethnic groups. This study aimed to investigate relationships between regional and WB fat depots and insulin sensitivity (IS) in White European (WE) and Black West African (BWA) men with T2D.

In a cross-sectional study, $17 \mathrm{WE}$ and $17 \mathrm{BWA}$ men with T2D underwent magnetic resonance imaging (MRI; Siemens 1.5-Tesla) from neck to knee to assess subcutaneous and VAT. MRI data was analysed by an automated quantitative method (Klarismo Ltd.) to determine WB and regional fat depots. IS was measured using a hyperinsulinaemic-euglycaemic clamp, presented as the glucose disposal rate $(\mathrm{M})$ divided by mean insulin concentration $(\mathrm{M} / \mathrm{I})$.

The ethnic groups were well matched for age, weight and BMI (all $p>0 \cdot 05$ ). There were no ethnic differences in IS (WE: 0.26 (IQR 0.19) $v s$ BWA: 0.26 (IQR 0.19) $\mathrm{mg} / \mathrm{m}^{2} \mathrm{BSA} \mathrm{min}^{-1} / \mathrm{pmol} / \mathrm{l}, p=0.524$ ), \% WB fat (WE: 30.2 (SD 6.0) $v s$ BWA: 26.4 (SD 5.8) \%, $p=0.070$ ), subcutaneous leg fat (WE: 2082 (SD 743) vs BWA: 2019 (SD 630) ml, $p=0.791$ ) and WB subcutaneous fat (WE: 13338 (IQR 6746) vs BWA: 12062 (IQR 6619) ml, $p=0.153$ ), however the BWA men had significantly lower WC (WE: 112 (SD 13.4) vs BWA: 103 (SD 8.6) cm, $p=0.031$ ), VAT (WE: 6769 (SD 2729) $v s$ BWA: 4132 (SD 1190) ml, $p=0.001$ ) and subcutaneous trunk fat (WE: 9378 (IQR 4531) vs BWA: 7660 (IQR 4378) $\mathrm{ml}, p=0.040$ ) compared to the WE men. IS was significantly inversely associated with WC and BMI as well as regional and WB adiposity in the WE men, whereas in the BWA men, significant inverse associations were only found with WB adiposity and subcutaneous fat of the left thigh (Table 1).

Table 1. Associations between insulin sensitivity (M/I) and adiposity measures in BWA and WE men

\begin{tabular}{llll}
\hline & & WE $(\mathrm{n}=17)$ & BWA $(\mathrm{n}=17)$ \\
\hline Waist circumference & R-value & $\mathbf{- 0 . 6 1 5 ^ { * * }}$ & -0.461 \\
Body mass index (BMI) & R-value & $\mathbf{- 0 . 6 1 6 ^ { * * }}$ & -0.415 \\
\% WB fat & R-value & $\mathbf{- 0 . 6 7 2 * *}$ & $\mathbf{- 0 . 4 8 5 *}$ \\
Subcutaneous fat of the left thigh & R-value & $\mathbf{- 0 . 7 0 1 * *}$ & $\mathbf{- 0 . 7 2 8 * *}$ \\
Subcutaneous fat of the trunk & R-value & $\mathbf{- 0 . 6 8 1 ^ { * * }}$ & -0.478 \\
WB subcutaneous fat & R-value & $\mathbf{- 0 . 6 9 6 * *}$ & $\mathbf{- 0 . 6 1 5 * *}$ \\
VAT & R-value & $\mathbf{- 0 . 4 8 3 *}$ & -0.373
\end{tabular}

Pearson's correlation analysis; significant correlations reported where: ${ }^{*} p<0 \cdot 05$, $* * p<0 \cdot 01$

Ethnic differences exist in the relationship between IS and WB and regional measures of adiposity that are linked to the metabolic syndrome and T2D, between men of WE and BWA ethnicity. The present study suggests that WB fat may be a better indication of increased risk of T2D in BWA men instead of the commonly used waist circumference.

1. Heymsfield SB, Peterson CM, Thomas DM et al. (2016) Obes Rev 17, 262-75.

2. DeBoer MD (2011) Expert Rev Endocrinol Metab 6, 279-89. 